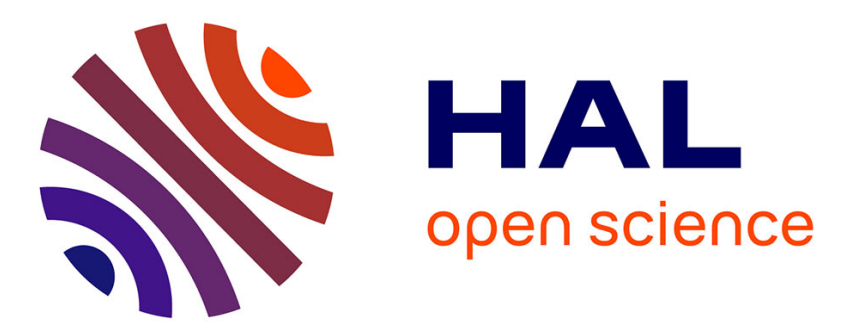

\title{
QoS-aware Power Allocation and Relay Placement in Green Cooperative FSO Communications
}

Ganesh Prasad, Deepak Mishra, Kamel Tourki, Ashraf Hossain, Merouane Debbah

\section{- To cite this version:}

Ganesh Prasad, Deepak Mishra, Kamel Tourki, Ashraf Hossain, Merouane Debbah. QoS-aware Power Allocation and Relay Placement in Green Cooperative FSO Communications. IEEE Wireless Communications and Networking Conference, Apr 2019, Marrakech, Morocco. hal-02089221

\section{HAL Id: hal-02089221 \\ https://hal-centralesupelec.archives-ouvertes.fr/hal-02089221}

Submitted on 3 Apr 2019

HAL is a multi-disciplinary open access archive for the deposit and dissemination of scientific research documents, whether they are published or not. The documents may come from teaching and research institutions in France or abroad, or from public or private research centers.
L'archive ouverte pluridisciplinaire HAL, est destinée au dépôt et à la diffusion de documents scientifiques de niveau recherche, publiés ou non, émanant des établissements d'enseignement et de recherche français ou étrangers, des laboratoires publics ou privés. 


\title{
QoS-aware Power Allocation and Relay Placement in Green Cooperative FSO Communications
}

\author{
Ganesh Prasad*, Deepak Mishra ${ }^{\dagger}$, Kamel Tourki ${ }^{\ddagger}$, Ashraf Hossain*, and Merouane Debbah ${ }^{\ddagger}$ \\ *Department of Electronics and Communication Engineering, National Institute of Technology, Silchar, India \\ ${ }^{\dagger}$ Department of Electrical Engineering (ISY), Linköping University, Sweden

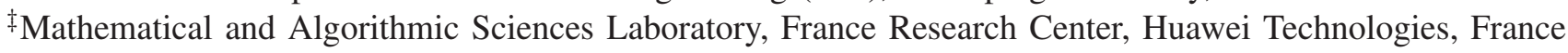

\begin{abstract}
Due to increasing quality-of-service (QoS) demand in already congested radio spectrum, there is a need for designing energy-efficient free space optical (FSO) communication networks. Considering a realistic fading model incorporating the fluctuations in angle-of-arrival, we minimize the outage probability for error free transmission of high data volumes through optimizing the power allocation (PA) and relay placement (RP) in a dual-hop decode-and-forward (DF) relay-assisted cooperative FSO communication with coherent detection and direct link unavailability. As this problem is nonconvex, first the optimal PA between source and relay is obtained using a global optimization algorithm. Also, a closed form for the solution is obtained using a tight analytical approximation with the assumption that atmospheric turbulence over both the links is nearly same. Next, we optimize the RP followed by the outage probability is jointly minimized using alternating optimization algorithm. Numerical results validate the outage analysis and provide key insights on optimal PA and RP yielding an outage enhancement of around $37 \%$ over the benchmark scheme.
\end{abstract}

\section{INTRODUCTION}

High data rate demand in the congested radio frequency band and need for green communication designs have enforced a research interest in free-space optical (FSO) networks [1]. The potential applications of FSO ranges from household applications to satellite communications [1]. Furthermore, as satellites will be eventually integrated with the $5 \mathrm{G}$ networks to offload high terrestrial traffic and reduce congestion in the backhaul networks [2], FSO frameworks can play a significant role there. However, the range of FSO link is limited by a lot of impairments caused by atmospheric turbulence, pointing error, and angle-of-arrival (AOA) fluctuations [3]. These limitations can be combated by reducing the link length using relayassisted cooperative FSO communications [4].

\section{A. Related Works}

A realistic FSO channel is investigated in [3], [5], [6], where the impairments due to AOA fluctuation are considered along with atmospheric turbulence and pointing error. In [5], experimental investigations are carried out for AOA and atmospheric turbulence, whereas closed form expressions are derived in [6] to compute the effect of spectral power law variations on the AOA fluctuations. Authors in [3] modeled the fading due to AOA fluctuation by including the atmospheric turbulence and transceiver vibrations, while highlighting the advantage of coherent detection on outage performance. However, these works did not investigate energy optimization in FSO networks.
In [7]-[9], various energy optimization techniques have been discoursed in cooperative FSO communications while considering only fading caused by atmospheric turbulence. A power allocation (PA) strategy over the source and relay nodes is described in [7] for minimizing the upper bound of frame error probability (FEP) over the network, whereas in [8], the power optimization in a dual-hop cooperative communication without direct link is investigated to minimize the average bit error probability (BEP). PA between source and relay nodes under delay quality of service $(\mathrm{QoS})$ constraints is evaluated in [9]. Also, note that in [7], [8], the signals are taken with intensity modulation and direct detection (IM/DD), whereas a coherent detection is used in [9]. Yet in a realistic channel with atmospheric turbulence, pointing error, and AOA fluctuations, joint optimization of PA and relay placement (RP) in a coherent network has not been investigated.

\section{B. Motivation and Key Contributions}

From the recent studies, there is a need for design of an energy-efficient network in presence of AOA fluctuations along with atmospheric turbulence and pointing error. In addition to it, the outage performance at FSO receiver can be significantly enhanced by coherent detection due to its special temporal selectivity, heterodyne gain, and noise rejection capability [10]. Also in a high data rate communications, a delay constraint bit error probability (BEP) based QoS metric gives essentially an error free transmission when forward error correction is applied. Therefore for fully exploiting these benefits in a realistic environment, we minimize the $Q o S$ aware outage probability by jointly optimizing the PA and $R P$ in a dual-hop decode-and-forward $(D F)$ relay-assisted coherent FSO communication without direct link availability. Although many works have been done in wireless networks for joint optimization of PA and RP, it is challenging in FSO communications as the fading due to atmospheric turbulence, pointing error, and AOA is entirely different from multipath fading.

The key contribution of this work is five-fold. (1) Outage expression based on BEP for a DF relay-assisted FSO network is obtained and a joint optimization problem is formulated to minimize it. (2) The optimization problem for optimal PA is shown to possess generalized convexity individually with respect to source and relay transmit power. Using it, a global optimization algorithm is presented which fastly converges to the optimal PA within an acceptable tolerance. (3) Analytical 
bounds for the feasible global optimal solution along with tight approximation for it are also proposed to gain nontrivial design insights. (4) Also, the global solution for an optimal $\mathrm{RP}$ is obtained with less complexity within an acceptable tolerence and corresponding joint optimization of PA and RP is evaluated using an alternting optimization algorithm. (5) The analysis is numerically validated via extensive simulations, providing the key insights on optimal PA and RP. Also, the achievable performance gains of different optimization methods are compared against a benchmark schemes.

\section{SySTEM MODEL}

\section{A. Network Topology}

In the discoursed dual-hop, half-duplex DF cooperative FSO network, the source $\mathcal{S}$ communicates with destination $\mathcal{D}$ via relay $\mathcal{R}$, where the nodes $\mathcal{R}$ and $\mathcal{D}$ are placed at $d_{\mathcal{S} \mathcal{R}}$ and $L$ respectively from $\mathcal{S}$. The direct $\mathcal{S}$-to- $\mathcal{D}$ link is assumed to be absent or its effect can be neglected due to blockage or fading loss [1]. For efficient utilization, the transmit power $P_{\mathcal{S}}$ and $P_{\mathcal{R}}$ of $\mathcal{S}$ and $\mathcal{R}$ respectively share a common power budget $P_{B}$. Both these nodes transmit the optical signals at operating wavelength $\lambda$ and are composed of single antenna for the transmission and reception [11].

\section{B. Channel Modeling}

As half-duplex is used in the cooperative dual-hop network, the transmission of source information from $\mathcal{S}$ to $\mathcal{D}$ takes place in two slots: first from $\mathcal{S}$ to $\mathcal{R}$ followed by from $\mathcal{R}$ to $\mathcal{D}$. The transmit signal over $i j \in\{\mathcal{S} \mathcal{R}, \mathcal{R} \mathcal{D}\}$ where $i j$ denotes $i$-to- $j$ link, faces three independent fading due to atmospheric turbulence, pointing error and AOA whose respective power gains are represented by $h_{i j}^{a}, h_{i j}^{p}$, and $h_{i j}^{a o a}$, respectively. We assume that the atmospheric turbulence is weak and the fading gain $h_{i j}^{a}$ has a log normal-distribution with probability density function (PDF) is given by [12, eq. (4)]. The pointing error occurs due to transmitter vibrations on irradiance where the PDF of $h_{i j}^{p}$ is expressed as in [3, eq. (15)]. Gain $h_{i j}^{a o a}$ due to AOA fluctuations is given by (1b) for coherent detection with perfect phasefront compensation [3]. The collective fading power gain of the channel is given as $h_{i j}=h_{i j}^{a} h_{i j}^{p} h_{i j}^{a o a}$. Joint cumulative density function (CDF) of $h_{i j}^{a}$ and $h_{i j}^{p}$, where $h_{i j}^{a p}=h_{i j}^{a} h_{i j}^{p}$ is given by (1a) [3].

$$
\begin{aligned}
& F_{i j}(x)=\operatorname{Pr}\left(h_{i j}^{a p} \leq x\right)=\frac{1}{2} \exp \left(r_{i j}^{2} \ln \frac{x}{A_{i j}^{0}}+2 \sigma_{i j}^{2} r_{i j}^{2}\right. \\
& \left.+2 \sigma_{i j}^{2} r_{i j}^{4}\right) \operatorname{erfc}\left(\frac{\ln \frac{x}{A_{i j}^{0}}+\mu_{i j}}{\sqrt{8} \sigma_{i j}}\right)+\frac{1}{2} \operatorname{erfc}\left(\frac{-\ln \frac{x}{A_{i j}^{0}}-2 \sigma_{i j}^{2}}{\sqrt{8} \sigma_{i j}}\right),
\end{aligned}
$$

$h_{i j}^{a o a}=\mathcal{W}\left(\sqrt{D_{i j}} \lambda\right)$,

where $\operatorname{erfc}(\cdot)$ is the complementary error function, $\mu_{i j}=$ $2 \sigma_{i j}^{2}\left(1+2 r_{i j}^{2}\right), \sigma_{i j}^{2}=1.06 \lambda^{-7 / 6} d_{i j}^{11 / 6} C_{i j}^{2}$ is the standard deviation of the log-amplitude fluctuations, $d_{i j}$ is the link length, $C_{i j}^{2}$ is refractive index structure constant, $r_{i j}=\frac{w_{i j}^{e q}}{2 \sigma_{i j}^{s}}$ is the ratio of the equivalent beam waist $w_{i j}^{e q}$ to the pointing error displacement standard deviation $\sigma_{i j}^{s}$ at the receiver, $A_{i j}^{0}=$ $\left[\operatorname{erf}\left(v_{i j}\right)\right]^{2}$ is the fraction of the received power in the absence of pointing error, $w_{i j}^{e q}=w_{i j} \sqrt{0.5 \sqrt{\pi} v_{i j}^{-1} \operatorname{erf}\left(v_{i j}\right) \exp \left(v_{i j}^{2}\right)}$, $v_{i j}=\frac{\sqrt{\pi} d}{2 \sqrt{2} w_{i j}}, w_{i j}$ is the beam waist, and $d$ is diameter of aperture of the receiver. In (1b), the gain $h_{i j}^{a o a}$ is obtained by coherent detection with perfect phasefront compensation. Here, $\mathcal{W}(\xi)=1-J_{0}^{2}\left(\frac{\pi \xi}{\lambda}\right)-J_{1}^{2}\left(\frac{\pi \xi}{\lambda}\right)$ in which $J_{0}$ and $J_{1}$ are the Bessel function of the first kind of order zero and one, respectively. The field-of-view (FOV) ratio $D_{i j}$ is the ratio between FOV and diffraction limited solid angles at receiver.

\section{PROBLEM DESIGN}

In this section, initially we analyzed the outage probability of a single-hop FSO network using which the expression for dual-hop communication is obtained.

\section{A. Outage Probability in a Single-hop}

As the channel coherence time in millisecond is long compared to the bit period of an optical signal with high data rate, a large number of bits can be distorted in a poor channel condition. Therefore, the outage probability based on error free transmission has been taken as a QoS metric. The outage probability of $i j$ link is defined as $P_{O}^{i j} \triangleq \operatorname{Pr}\left(P_{e}^{i j}>P_{e}^{t h}\right)$ which is the probability that BEP $P_{e}^{i j}$ of $i j$ link is greater than the threshold BEP $P_{e}^{t h}$. The BEP $P_{e}^{i j}$ at the coherent receiver of the link is given as [3, eq. (41)]:

$$
P_{e}^{i j}=\frac{1}{2} \exp \left(-\frac{2 h_{i j}^{a p} \mathcal{W}\left(\sqrt{D_{i j}} \lambda\right) N_{i j}^{s}}{1+\mathcal{W}\left(\sqrt{D_{i j}} \lambda\right) N_{i j}^{n}}\right),
$$

where $N_{i j}^{s}=\frac{\tau P_{i} \lambda}{c h_{p k}}$ is number transmit photons in a bit interval $\tau, P_{i}$ is the transmit power by node $i \in\{\mathcal{S}, \mathcal{R}\}, h_{p k}$ is Plank's constant, $c$ is the speed of light, and $N_{i j}^{n}$ is the background noise photon counts in a bit interval. Using (2), $P_{O}^{i j}$ can be expressed in terms of CDF $F_{i j}(x)$ as given in (1a) by taking all the parameters of $P_{e}^{i j}$ on right side except $h_{i j}^{a p}$ as:

$$
P_{O}^{i j}=\operatorname{Pr}\left(P_{e}^{i j}>P_{e}^{t h}\right)=\operatorname{Pr}\left(h_{i j}^{a p}<\mathfrak{G}_{i j}\right)=F_{i j}\left(\mathfrak{G}_{i j}\right),
$$

where $\mathfrak{G}_{i j}=\left(1+\mathcal{W}\left(\sqrt{D_{i j}} \lambda\right) N_{i j}^{n}\right) \ln \frac{1}{2 P_{e}^{t h}}\left[2 \mathcal{W}\left(\sqrt{D_{i j}} \lambda\right) N_{i j}^{s}\right]^{-1}$ for $i j \in\{\mathcal{S R}, \mathcal{R} \mathcal{D}\}$. Using (3), next we obtain the expression of outage probability for the dual-hop FSO network.

\section{B. Outage Analysis in a Dual-hop DF Network}

To obtain an error free transmission in the dual-hop network, we need to minimize the end-to-end outage probability based on BEP similar to the case of single-hop communication. Performance of the dual-hop DF relay-assisted cooperative FSO communication without direct link is bottle-necked by the weaker link. Considering QoS-aware communication with BEP as the QoS metric, the bottleneck link which gives the higher BEP, determines the outage probability $p_{\text {out }}$ of the dualhop communication in (4a) as:

$$
\begin{aligned}
& p_{\text {out }}=\operatorname{Pr}\left[\max \left\{P_{e}^{\mathcal{S R}}, P_{e}^{\mathcal{R} \mathcal{D}}\right\}>P_{e}^{t h}\right] \\
& =1-\left(1-\operatorname{Pr}\left[P_{e}^{\mathcal{S R}}>P_{e}^{t h}\right]\right)\left(1-\operatorname{Pr}\left[P_{e}^{\mathcal{R D}}>P_{e}^{t h}\right]\right) \\
& =F_{\mathcal{S R}}\left(\mathfrak{G}_{\mathcal{S R}}\right)+F_{\mathcal{R D}}\left(\mathfrak{G}_{\mathcal{R D}}\right)-F_{\mathcal{S R}}\left(\mathfrak{G}_{\mathcal{S R}}\right) F_{\mathcal{R D}}\left(\mathfrak{G}_{\mathcal{R D}}\right) \\
& =P_{O}^{\mathcal{S R}}+P_{O}^{\mathcal{R D}}-P_{O}^{\mathcal{S R}} P_{O}^{\mathcal{R} \mathcal{D}}
\end{aligned}
$$


In (4b), $p_{\text {out }}$ is expressed in terms of the outage probability of the individual link by taking the assumption that $\mathcal{S R}$ and $\mathcal{R D}$ links are independent of each other. Using (3) with some algebraic simplification, $p_{\text {out }}$ can be expressed in CDF of underlying variable of each link by (4c) which can be further expressed as in (4d) using (3). Next, we formulate a joint optimization problem to minimize $p_{\text {out }}$ by optimizing the PA and RP over the dual-hop network.

\section{Problem Definition}

The optimization problem for minimizing $p_{\text {out }}$ for a given power budget $P_{B}$ can be expressed as:

$$
\begin{aligned}
& \text { (P0): } \underset{P_{\mathcal{S}}, P_{\mathcal{R}}, d_{\mathcal{S} \mathcal{R}}}{\operatorname{minimize}} p_{\text {out }} \text {, } \\
& \text { subject to } C 1: P_{\mathcal{S}}, P_{\mathcal{R}} \geq 0 \text {, } \\
& C 3: d_{\mathcal{S R}} \geq \delta \text {, } \\
& C 2: P_{\mathcal{S}}+P_{\mathcal{R}} \leq P_{B}, \\
& C 4: d_{\mathcal{S R}} \leq L-\delta \text {, }
\end{aligned}
$$

where $C 1$ and $C 2$ are power positivity and power budget constraints, respectively whereas $C 3$ and $C 4$ are constraints on the RP. $\delta$ is the minimum distance between the two nodes over the network. As the objective of the problem (P0) is nonconvex function of $P_{\mathcal{S}}, P_{\mathcal{R}}$, and $d_{\mathcal{S R}}$, it requires investigation of an alternate approach for the optimal solution. To obtain the solution, first we investigate the problem for individual optimization of PA and RP, then it is analyzed jointly.

\section{OPTIMAL PA FOR A FIXED RP}

Using (P0), the optimal PA over $\mathcal{S}$ and $\mathcal{R}$ for given $\mathrm{RP} d_{\mathcal{S} \mathcal{R}}$ can be obtained by minimizing $p_{\text {out }}$ through optimizing $P_{\mathcal{S}}$ and $P_{\mathcal{R}}$ under the constraint of $C 1$ and $C 2$. The conditional generalized convexity of the problem in two variables is described as follows.

\section{A. Generalized Convexity}

Although the problem is nonconvex, end-to-end outage probability $p_{\text {out }}$ is pseudolinear as it is monotonically decreasing in both $P_{\mathcal{S}}$ and $P_{\mathcal{R}}$ because the underlying outage probability for each link is respectively decreasing in their transmit powers $P_{\mathcal{S}}$ and $P_{\mathcal{R}}$. Therefore, if we optimize the problem for individual $P_{\mathcal{S}}$ (or $P_{\mathcal{R}}$ ) for a given $P_{\mathcal{R}}$ (or $P_{\mathcal{S}}$ ), it becomes a generalized convex problem due to pseudolinear objective function $p_{\text {out }}$ along with linear box constraints [13]. But in case of joint optimization of $P_{\mathcal{S}}$ and $P_{\mathcal{R}}$, the problem is not a generalized convex. So, we investigate the region where it is generalized convex and the optimal solution is obtained by golden search [14], whereas in a nonconvex regions the optimal point is evaluated by full linear search.

Since $p_{\text {out }}$ is monotonically decreasing with both $P_{\mathcal{S}}$ and $P_{\mathcal{R}}$, the power budget $P_{B}$ is fully utilized. Thus, we can eliminate $P_{\mathcal{R}}$ in the expression of $p_{\text {out }}$ by substituting $P_{\mathcal{R}}=P_{B}-P_{\mathcal{S}}$ which can be expressed as $\widehat{p_{\text {out }}}\left(P_{\mathcal{S}}, d_{\mathcal{S R}}\right)=$ $\left\{\left.p_{\text {out }}\right|_{P_{\mathcal{R}}=P_{B}-P_{\mathcal{S}}}\right\}$. For obtaining the potential region for the optimal solution, first we investigate the expression of $p_{\text {out }}$. As $p_{\text {out }}$ in $(4 \mathrm{~d})$ can be shown as $p_{\text {out }}=1-\left(1-P_{O}^{\mathcal{S} \mathcal{R}}\right)\left(1-P_{O}^{\mathcal{R} D}\right)$, first we find the common region where $\left(1-P_{O}^{\mathcal{S R}}\right)$ and $\left(1-P_{O}^{\mathcal{R} D}\right)$ are concave with respect to $P_{\mathcal{S}}$. If we consider $P_{O}^{\mathcal{S R}}$, it is $\mathrm{CDF}$ of variable $\mathfrak{G}_{\mathcal{S} \mathcal{R}}$ (cf. (3)) and its first derivative is PDF which is unimodal with respect to underlying variable [15]. Although the PDF is always positive, initially its rate of increment increases then it decreases. It implies that $P_{O}^{\mathcal{S R}}$ is convex followed by concave in nature. As $P_{\mathcal{S}}$ is reciprocal of $\mathfrak{G}_{\mathcal{S R}}$, the characteristics of $P_{O}^{\mathcal{S} \mathcal{R}}$ and $\left(1-P_{O}^{\mathcal{S} R}\right)$ changes from concave to convex and convex to concave respectively with $P_{\mathcal{S}}$. Using the same analogy it can be observed that the nature of $\left.\left(1-P_{O}^{\mathcal{R} D}\right)\right|_{P_{\mathcal{R}}=P_{B}-P_{\mathcal{S}}}$ changes from concave to convex with $P_{\mathcal{S}}$. Therefore the upper bound $P_{t h}^{U}$ and lower bound $P_{t h}^{L}$ of the common region where $\left(1-P_{O}^{\mathcal{S} \mathcal{R}}\right)$ and $\left(1-P_{O}^{\mathcal{R} \mathcal{D}}\right)$ are concave are expressed as:

$$
\begin{aligned}
& P_{t h}^{L}=\max \left\{0,\left\{P_{\mathcal{S}} \mid \frac{\partial^{2}\left(1-P_{O}^{\mathcal{S R}}\right)}{\partial P_{\mathcal{S}}^{2}}=0\right\}\right\} \\
& P_{t h}^{U}=\min \left\{\left\{P_{\mathcal{S}}\left|\frac{\partial^{2}\left(1-P_{O}^{\mathcal{R} D}\right)}{\partial P_{\mathcal{S}}^{2}}\right|_{\left.\left.P_{\mathcal{R}}=P_{B}-P_{\mathcal{S}}=0\right\}, P_{B}\right\},}\right.\right.
\end{aligned}
$$

where the double derivatives have unique solution due to one time change in characteristics of $\left(1-P_{O}^{\mathcal{S} \mathcal{R}}\right)$ and $\left(1-P_{O}^{\mathcal{R} \mathcal{D}}\right)$ with $P_{\mathcal{S}}$. As the product of two concave is pseudoconcave [13, Table 5.1], $p_{\text {out }}$ which is negative of the product is pseudoconvex. So, the problem is generalized convex in the region $\left(P_{t h}^{L}, P_{t h}^{U}\right)$, whereas it is nonconvex in the regions $\left(0, P_{t h}^{L}\right)$ and $\left(P_{t h}^{U}, P_{B}\right)$ for $0 \leq P_{\mathcal{S}} \leq P_{B}$. Using the obtained potential disjoint regions, we compute the optimal solution of nonconvex problem through a global optimization algorithm as follows.

\section{B. $\epsilon$-Global Optimization Algorithm}

From Section IV-A, the optimization problem is generalized convex in the region $\left(P_{t h}^{L}, P_{t h}^{U}\right)$ and nonconvex in $\left(0, P_{t h}^{L}\right)$ and $\left(P_{t h}^{U}, P_{B}\right)$. So using Algorithm 1, three sub-optimal solutions can be obtained within an acceptable tolerance $\epsilon$ by applying golden search in $\left(P_{t h}^{L}, P_{t h}^{U}\right)$ and full linear search in remaining two regions by varying $P_{\mathcal{S}}$, where $\mathcal{X}_{t h}^{L}=P_{t h}^{L}, \mathcal{X}_{t h}^{U}=P_{t h}^{U}$, $\mathcal{X}_{L}=0$, and $\mathcal{X}_{U}=P_{B}$. One of the sub-optimal solution is set to global at which $\widehat{p_{\text {out }}}$ achieves its minimum value.

Total number of steps involved in obtaining the optimal solution within an acceptable tolerance $\epsilon$ is given by $\frac{P_{B}+P_{t h}^{L}-P_{t h}^{U}}{\epsilon}+2 \ln \left(\frac{P_{t h}^{U}-P_{t h}^{L}}{\epsilon}\right)+4 \ln \left(\frac{P_{B}}{\epsilon}\right)$, where the first and second terms represent the number of steps required for the optimal solution in two linear and one golden search respectively, whereas the third term account for the evaluation of two thresholds $P_{t h}^{L}$ and $P_{t h}^{U}$ with tolerance $\epsilon$. Note that the number of steps decreases significantly with increment of $P_{t h}^{U}$ or with decrement of $P_{t h}^{L}$, because the region for golden search over $\left(P_{t h}^{L}, P_{t h}^{U}\right)$ increases while the other two regions for linear search decrease. Numerically, it has been observed as in Figs. 4 and 5 that the two thresholds lie near to boundary of the region $\left(0, P_{B}\right)$ which diminish the computational complexity due to linear search. Thus, practically the optimal solution can be obtained by applying golden search over the whole region with significantly less computational complexity $2 \ln \left(\frac{P_{B}}{\epsilon}\right)$. Further, conditionally a closed form of the optimal solution is obtained through analytical approximation which is described below. 
$\overline{\text { Algorithm } 1 \text { Computation of optimal value of } \mathcal{X} \in\left\{P_{\mathcal{S}}, d_{\mathcal{S R}}\right\}}$ within an acceptable tolerance $\epsilon$.

Input: $\epsilon$, upper bound $\mathcal{X}_{U}$, and lower bound $\mathcal{X}_{L}$

Output: Optimal value $\mathcal{X}^{*}$

1: Compute the upper threshold $\mathcal{X}_{t h}^{U}$ and lower threshold $\mathcal{X}_{t h}^{L}$ using (5) or (7).

2: For $\mathcal{X}_{t h}^{L} \leq \mathcal{X} \leq \mathcal{X}_{t h}^{U}$, compute $\mathcal{X}_{1}^{*}=\underset{\mathcal{X}}{\operatorname{argmin}} \widehat{p_{\text {out }}}$ using golden search within an acceptable tolerance $\epsilon$.

3: Evaluate $\mathcal{X}_{2}^{*}=\underset{\mathcal{X}}{\operatorname{argmin}} \widehat{p_{\text {out }}}$ through linear search by varying $\mathcal{X}$ with step size $\epsilon \stackrel{\mathcal{X}}{\text { over the region }}\left(\mathcal{X}_{L}, \mathcal{X}_{t h}^{L}\right)$.

4: Again similar to step 3, find the optimal value $\mathcal{X}_{3}^{*}$ using the linear search over the region $\left(\mathcal{X}_{t h}^{U}, \mathcal{X}_{U}\right)$.

5: Find $\mathcal{X}^{*}=\operatorname{argmin} \widehat{p_{\text {out }}}$ for $i \in\{1,2,3\}$. $\left\{\mathcal{X}_{i}\right\}$

\section{Analytical Approximation}

In a DF relay-assisted dual-hop cooperative FSO communication, the optimal PA between $\mathcal{S}$ and $\mathcal{R}$ attempts to minimize the outage by balancing the trade-off between the quality of the $\mathcal{S R}$ and $\mathcal{R D}$ links. And, the optimal point is reached when outage probability of both the links becomes equal. From (3), outage probability of a link depends on joint CDF of power gain of atmospheric turbulence and pointing error. With the assumption that atmospheric turbulence of both the links are same which make the distribution of both the links approximately same, the outage probability of both the links becomes equal by making the mean value of underlying random variable equal. Therefore, $P_{O}^{\mathcal{S R}}=P_{O}^{\mathcal{R D}} \Rightarrow F_{\mathcal{S R}}\left(\mathfrak{G}_{\mathcal{S R}}\right)=F_{\mathcal{R D}}\left(\mathfrak{G}_{\mathcal{R D}}\right)$ can be achieved by $\frac{\mathbb{E}\left[h_{\mathcal{S} \mathcal{R}]}^{a} \mathbb{E}\left[h_{\mathcal{S} \mathcal{R}}^{p}\right]\right.}{\mathfrak{b}_{2} \mathcal{S} \mathcal{S}}=\frac{\mathbb{E}\left[h_{\mathcal{R} \mathcal{D}}^{a}\right] \mathbb{E}\left[h_{\mathcal{R} \mathcal{D}}^{p}\right]}{\mathfrak{G}_{\mathcal{R} \mathcal{D}}}$, where $\mathbb{E}\left[h_{i j}^{a}\right]=1$ and $\mathbb{E}\left[h_{i j}^{p}\right]=\frac{r_{i j}^{2} A_{i j}}{r_{i j}^{2}+1}$ [3], [12]. Further, after some algebraic simplification the approximate optimal solution can be expressed as:

$$
\widehat{P_{\mathcal{S}}^{*}}=\left(\frac{\mathfrak{C}_{\mathcal{R D}}}{\mathfrak{C}_{\mathcal{S R}}+\mathfrak{C}_{\mathcal{R D}}}\right) P_{B}
$$

where $\mathfrak{C}_{i j}=\frac{r_{i j}^{2} A_{i j} \mathcal{W}\left(\sqrt{D_{i j}} \lambda\right)}{\left(r_{i j}^{2}+1\right)\left[1+\mathcal{W}\left(\sqrt{D_{i j}} \lambda\right) N_{i j}^{n}\right]}$; for $i j \in\{\mathcal{S R}, \mathcal{R} \mathcal{D}\}$. Using (6), the approximate optimal power $\widehat{P_{\mathcal{R}}^{*}}$ at $\mathcal{R}$ can be computed as $\widehat{P_{\mathcal{R}}^{*}}=P_{B}-\widehat{P_{\mathcal{S}}^{*}}$. In Section VII, we have shown in Fig. 3 that the approximate solution yields a tight analytical approximation of the obtained optimal solution using Algorithm 1.

\section{OPTIMAL RP FOR A FIXED PA}

For a given PA, the optimal RP is achieved by minimizing $\widehat{p_{\text {out }}}$ under the constraint of $C 3$ and $C 4$. Since the problem is nonconvex, we evaluate the potential regions for the optimal solution similar to the case for evaluation of optimal PA as discoursed in Section IV-A. From $(4 \mathrm{~b}), \widehat{p_{\text {out }}}\left(P_{\mathcal{S}}, d_{\mathcal{S R}}\right)=$ $\left\{\left.p_{\text {out }}\right|_{P_{\mathcal{R}}=P_{B}-P_{\mathcal{S}}}\right\}$ is pseudoconvex function of $d_{\mathcal{S} \mathcal{R}}$ when $\left(1-P_{O}^{\mathcal{S R}}\right)$ and $\left(1-P_{O}^{\mathcal{R D}}\right)$ are concave with respect to $d_{\mathcal{S R}}$ in a common region. The upper bound $d_{t h}^{U}$ and lower bound $d_{t h}^{L}$ of the common region are computed as:

$$
\begin{aligned}
& d_{t h}^{U}=\min \left\{\left\{d_{\mathcal{S R}} \mid \frac{\partial^{2}\left(1-P_{O}^{\mathcal{S R}}\right)}{\partial d_{\mathcal{S R}}^{2}}=0\right\}, L-\delta\right\} \\
& d_{t h}^{L}=\max \left\{\delta,\left\{d_{\mathcal{S R}} \mid \frac{\partial^{2}\left(1-P_{O}^{\mathcal{R} D}\right)}{\partial d_{\mathcal{S R}}^{2}}=0\right\}\right\}
\end{aligned}
$$

It entails that the problem is generalized convex over the region $\left(d_{t h}^{L}, d_{t h}^{U}\right)$ and is nonconvex in the regions $\left(\delta, d_{t h}^{L}\right)$ and $\left(d_{t h}^{U}, L-\delta\right)$. Using Algorithm 1, one sub-optimal solution is obtained in the region $\left(d_{t h}^{L}, d_{t h}^{U}\right)$ using golden search, whereas the other two sub-optimal solutions are obtained using linear search in $\left(\delta, d_{t h}^{L}\right)$ and $\left(d_{t h}^{U}, L-\delta\right)$, where $\mathcal{X}_{L}=\delta, \mathcal{X}_{U}=L-\delta$, $\mathcal{X}_{t h}^{L}=d_{t h}^{L}$, and $\mathcal{X}_{t h}^{U}=d_{t h}^{U}$. One of the solution is set to global at which $\widehat{p_{\text {out }}}$ achieves its minimum value. Numerically, it has been investigated that the two thresholds $d_{t h}^{L}$ and $d_{t h}^{U}$ mostly lie near to boundary of the region $(\delta, L-\delta)$ which is also depicted in Figs. 6 and 7. Thus, the optimal RP can be obtained by applying golden search over the whole region with computational complexity $2 \ln \left(\frac{L-2 \delta}{\epsilon}\right)$.

\section{Joint Minimization of $\widehat{p_{\text {out }}}$}

The joint optimization of PA and RP is obtained by evaluating the problem (P0) under the constraint of $\mathrm{C} 1, \mathrm{C} 2, \mathrm{C} 3$, and $\mathrm{C} 4$. In this case, the joint optimal point $\left(P_{\mathcal{S}}^{*}, d_{\mathcal{S} \mathcal{R}}^{*}\right)$ is obtained using alternating optimization algorithm as described in Algorithm 2.

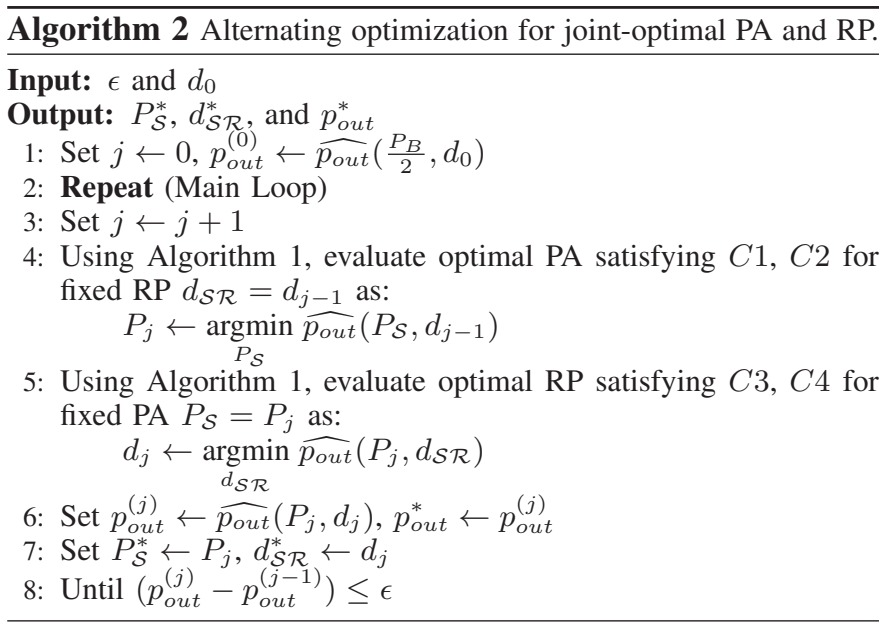

The algorithm begins with an initial point $d_{0}=\frac{L}{2}$ and repetitively gives the alternating minimization sequence of PA and RP until $\left(p_{\text {out }}^{(j)}-p_{\text {out }}^{(j-1)}\right) \leq \epsilon$. The sequence $p_{\text {out }}^{(j)}$ is decreasing and converges fast to jointl-optimal point $\left(P_{\mathcal{S}}^{*}, d_{\mathcal{S} \mathcal{R}}^{*}\right)$.

\section{NUMERICAL RESULTS}

In this section, we conduct a numerical investigation on the proposed outage analysis and solution methodology. Unless otherwise stated the default system parameters are set as [3]: operating wavelength $\lambda=1550 \mathrm{~nm}, L=2 \mathrm{~km}, d_{\mathcal{S R}}=1 \mathrm{~km}$, $\delta=1 \mathrm{~m}, d=5 \mathrm{~cm}, w_{z}=0.5 \mathrm{~m}$, bit duration $\tau=1 \times 10^{-9}$ s, background noise $N_{i j}^{n}=1, P_{e}^{t h}=1 \times 10^{-4}, P_{B}=10$ $\mu W, P_{\mathcal{S}}=5 \mu W, \epsilon=1 \times 10^{-7}, \sigma_{i j}^{s}=0.1, D_{i j}=2$, and $C_{i j}^{2}=5 \times 10^{-14} \mathrm{~m}^{-2 / 3}$ for $i j \in\{\mathcal{S} \mathcal{R}, \mathcal{R} \mathcal{D}\}$.

Using Fig. 1, first we validate the expression of outage probability $\widehat{p_{\text {out }}}$ as given in (4). The simulation results are evaluated by first probing $10^{5}$ random realization of fading power gains due to atmospheric turbulence and pointing error in the two links for the corresponding maximum of the BEP of the two links to be greater than $10^{-4}$. After that the fraction, for which maximum of the BEP $>10^{-4}$ over the all random 


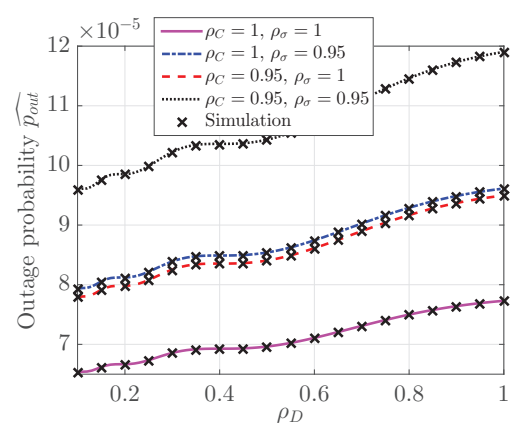

Fig. 1: $\widehat{p_{\text {out }}}$ with variation in channel conditions of the two links.

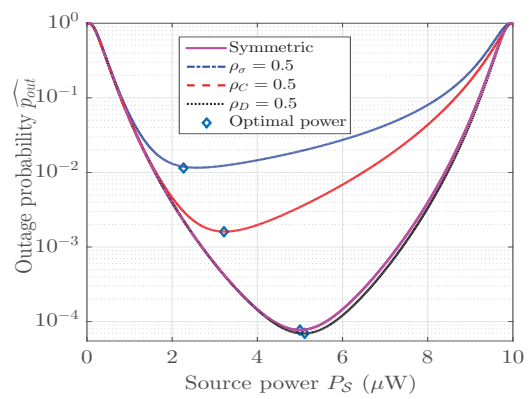

Fig. 2: $\widehat{p_{\text {out }}}$ vs $P_{\mathcal{S}}$ with various insights on optimal PA.

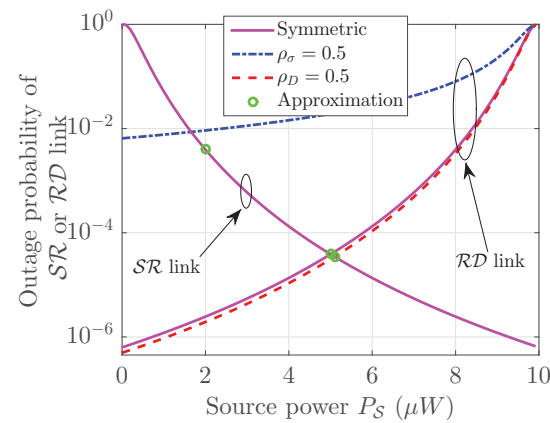

Fig. 3: Joint variation of outage probabilities for $\mathcal{S} \mathcal{R}$ and $\mathcal{R} \mathcal{D}$ links.

realizations, is computed as outage probability. The plot is obtained with relative fading gains caused by asymmetric atmospheric turbulence, pointing error, and AOA over $\mathcal{S R}$ and $\mathcal{R D}$ links represented by the ratios $\rho_{C}=\frac{C_{\mathcal{S}}^{2}}{C_{\mathcal{R}}^{2}}, \rho_{\sigma}=\frac{\sigma_{\mathcal{S}}^{s}}{\sigma_{\mathcal{R}}^{s}}$, and $\rho_{D}=\frac{D_{\mathcal{S R}}}{D_{\mathcal{R} D}}$, respectively. The variation of these ratios from 0.1 to 1 by varying the parameters of $\mathcal{R D}$ link while keeping the $\mathcal{S R}$ link at its default value. For the change in ratio $\rho_{D}$ from 0.1 to 0.5 , average increment in $\widehat{p_{\text {out }}}$ is 0.56 times of the increment for the change in $\rho_{D}$ from 0.5 to 1 . It implies that after certain point there is no significant improvement in outage performance due to large FOV.

For different channel impairments, the optimal PA at which $\widehat{p_{\text {out }}}$ achieves its minimum value has been described in Fig. 2 . The plot for $\widehat{p_{\text {out }}}$ vs $P_{\mathcal{S}}$ is obtained by varying one of the ratios while keeping other two equal to 1 . In symmetric network where all the three ratios are equal to 1 , optimally equal power is allocated to both the links which gives $P_{\mathcal{S}}^{*}=\frac{P_{B}}{2}=5$ $\mu W$. In the plot, for $\rho_{\sigma}=0.5$, the minimized $\widehat{p_{\text {out }}}$ is higher than $\rho_{C}=0.5$ which entails that pointing error deteriorates the outage more than atmospheric turbulence where higher power is allocated to $\mathcal{R D}$ than $\mathcal{S R}$ link. For $\rho_{D}=0.5$, the marginal gain in outage performance is achieved compared to the symmetric network.

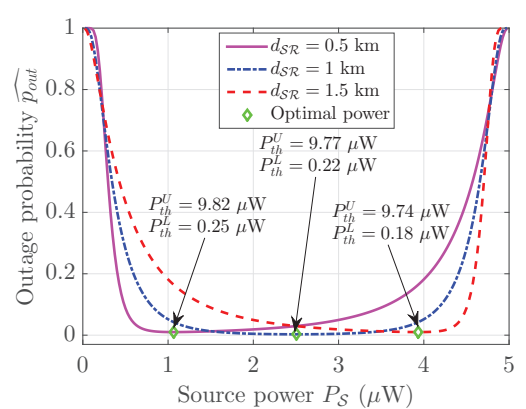

Fig. 4: Variation of $\widehat{p_{\text {out }}}$ with $P_{\mathcal{S}}$ for different relay positions.

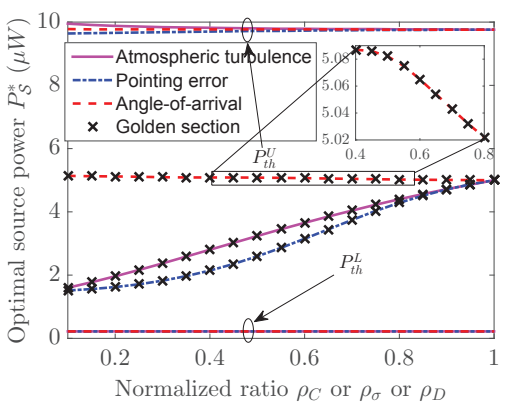

Fig. 5: Optimal PA as well as the validation of fast convergence of the global algorithm with different channel conditions.

For the obtained numerical optimal source power $P_{\mathcal{S}}^{*}$ in Fig. 2, a closed form insight given in (6) is validated through Fig. 3 where the atmospheric turbulence of both the links are assumed to be same as described in Section IV-C, i.e., $\rho_{C}=1$. Here the joint variation of outage probability of $\mathcal{S R}$ and $\mathcal{R D}$ links with $P_{\mathcal{S}}$ is obtained for different normalized ratios. The optimal point $P_{\mathcal{S}}^{*}$ obtained in Fig. 2 for symmetric, $\rho_{\sigma}=0.5$, and $\rho_{D}=0.5$ is $5.00,2.26$ and $5.11 \mu \mathrm{W}$ and corresponding optimal point evaluated using analytical approximation is $5.00,2.61$, and $5.12 \mu \mathrm{W}$, respectively. Therefore, the obtained approximate optimal points are tight approximation of the optimal solution evaluated using Algorithm 1 which are lying near to cross over points where the outage probability of the two links becomes equal.

Variation of $\widehat{p_{\text {out }}}$ with $P_{\mathcal{S}}$ for different relay positions has been shown in Fig. 4 where power budget $P_{B}=5 \mu \mathrm{W}$. It can be observed that optimal PA at source $\mathcal{S}$ is less when relay $\mathcal{R}$ is located near to source $\mathcal{S}$ and vice versa and for $d_{\mathcal{S} \mathcal{R}}^{*}=L / 2=$ $1 \mathrm{~km}, P_{\mathcal{S}}^{*}=P_{\mathcal{R}}^{*}=P_{B} / 2=2.5 \mu \mathrm{W}$. In Fig. 5, variation of $P_{\mathcal{S}}^{*}$ with atmospheric turbulence, pointing error and AOA of the two links which can be realized by varying $\rho_{C}, \rho_{\sigma}$, and $\rho_{D}$, respectively. Note that while varying one of the ratio, the other two are kept at unity. In the plot, it can be observed that with $\rho_{C}$ and $\rho_{\sigma}, P_{\mathcal{S}}^{*}$ increases at higher rate due to faster relative degradation of $\mathcal{S R}$ than $\mathcal{R} \mathcal{D}$ link. In case of $\rho_{D}$, the $\mathcal{S} \mathcal{R}$ link relatively improves at slower rate. Also, the plot depicts that the optimal solution obtained from Algorithm 1 is same as it is evaluated by applying golden search over the whole region $\left(0, P_{B}\right)$ because the thresholds $P_{t h}^{L}$ and $P_{t h}^{U}$ are lying near to the boundary of the region $\left(0, P_{B}\right)$ with $0.046 \%$ gap. Thus as discoursed in Section IV-B, the global solution can be obtained by applying golden search over the whole region which significantly reduces the computational complexity.

The variation of $\widehat{p_{\text {out }}}$ with relay location $d_{\mathcal{S R}}$ has been 


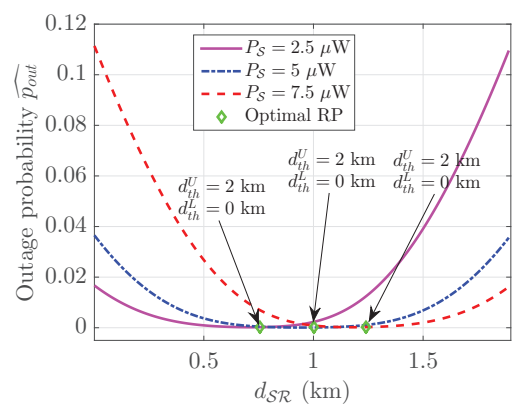

Fig. 6: Variation of $\widehat{p_{\text {out }}}$ with $d_{\mathcal{S R}}$ for different values of $P_{\mathcal{S}}$

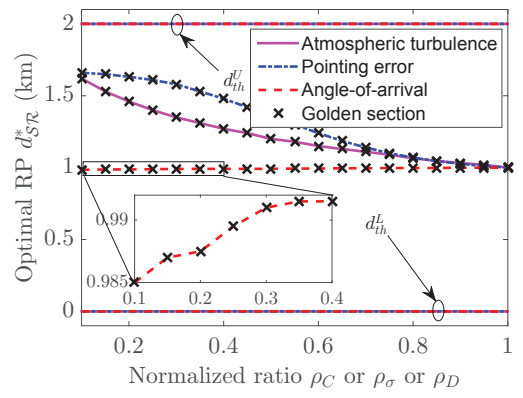

Fig. 7: Optimal RP as well as the validation of fast convergence of the global algorithm with different channel conditions.

depicted in Fig. 6. It shows that for higher allocation of $P_{\mathcal{S}}$, node $\mathcal{R}$ is optimally located near to destination node $\mathcal{D}$ and vice versa and for equal PA over $\mathcal{S}$ and $\mathcal{R}, \mathcal{R}$ is optimally located at the middle of $\mathcal{S}$ and $\mathcal{D}$ nodes. In Fig. 7, the variation of optimal RP is analyzed by varying one of the normalized ratio while keeping the other two fixed at unity. It can be observed that with $\rho_{C}$ and $\rho_{\sigma}$, the optimal RP decreases because of $\mathcal{S R}$ link is relatively degrades which requires lesser link length for the compensation. Whereas with variation of $\rho_{D}, d_{\mathcal{S} \mathcal{R}}^{*}$ increases marginally due to relative degradation of $\mathcal{R D}$ link at slower rate. Also, it can be realized that optimal solution obtained though Algorithm 1 is same as computed through applying golden search over the whole region $(0.001$ $\mathrm{km}, 1.999 \mathrm{~km})$ as $d_{t h}^{L}$ and $d_{t h}^{U}$ lie near to boundary with $0.012 \%$ gap. Thus as discoursed in Section V, the global solution for the optimal RP can be obtained simply by applying golden search over the whole region $(\delta, L-\delta)$.

In Fig. 8, different optimization schemes are compared against a fixed benchmark scheme where equal power is allocated to both the links. Percentage improvement is measured by varying one of the normalized ratio while keeping remaining two at unity. With variation of $\rho_{C}$ and $\rho_{D}$, optimal RP is competing the joint optimization, whereas with respect to $\rho_{\sigma}$ the optimal PA has better performance than optimal RP. It occurs because the atmospheric turbulence highly depends on the transmission distance compared to the pointing error. Average percentage improvement obtained through optimal PA, optimal RP, and joint optimization over benchmark scheme are $19.62 \%, 34.20 \%$, and $37.10 \%$, respectively.

\section{CONCLUding REMARKS}

We have efficiently solved the nonconvex joint optimization problem for the minimization on QoS-aware outage probability

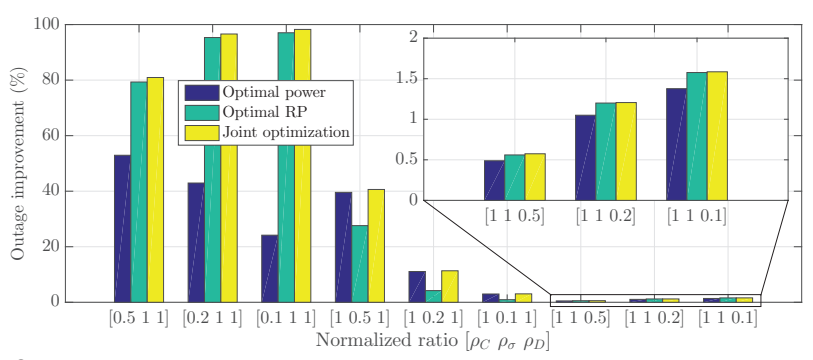

Fig. 8: Percentage improvement of different optimization schemes.

of the dual-hop network through a global optimization algorithm which obtains the optimal solutions within an acceptable tolerance. Initially, we minimize it by individually optimizing the PA and RP while keeping the other fixed. Thereafter, the joint solution is obtained using alternating optimization algorithm. In case of optimization of power control, we find the analytical insights on the optimal PA by an analytical approximation. Through the obtained numerical results, we validate the analysis and obtained the key insights on the optimal PA and RP. Practically, we realize that the optimal solution obtained through golden search over the whole region gives the same performance as global solution evaluated using global optimization algorithms.

\section{REFERENCES}

[1] I. K. Son and S. Mao, "A survey of free space optical networks," Digital Commun. and Networks, Elsevier, vol. 2, no. 3, pp. 67-77, 2017.

[2] J. Liu et al., "Joint placement of controllers and gateways in SDNenabled 5g-satellite integrated network," IEEE J. on Sel. Areas Commun. vol. 36, no. 2, pp. 221-232, Feb. 2018.

[3] S. Huang and M. Safari, "Free-space optical communication impaired by angular fluctuations," IEEE Trans. Wireless Commun., vol. 16, no. 11, pp. 7475-7487, Nov. 2017.

[4] G. K. Varotsos et al., "Outage performance of mixed, parallel and serial DF relayed FSO links over weak turbulence channels with nonzero boresight pointing errors," in Proc. IEEE Int. Conf. on Modern Circuits and Sys. Technologies, Thessaloniki, Greece, May 2018, pp. 1-4.

[5] A. Consortini, Y. Y. Sun, C. Innocenti, and Z. P. Li, "Measuring inner scale of atmospheric turbulence by angle of arrival and scintillation," Optics commun., vol. 216, no. 1-3, pp. 19-23, Feb. 2003.

[6] W. Du et al., "Angle-of-arrival fluctuations for wave propagation through non-kolmogorov turbulence," Optics Commun., vol. 282, no. 5, pp. 705708, Mar. 2009.

[7] C. Abou-Rjeily and S. Haddad, "Inter-relay cooperation: A new paradigm for enhanced relay-assisted FSO communications," IEEE Trans. Commun., vol. 62, no. 6, pp. 1970-1982, Jun. 2014.

[8] Z. G. Sun, H. Yu, and Y. J. Zhu, "A superimposed relaying strategy and power allocation for outdoor visible light communications," IEEE Access, vol. 5, pp. 9555-9561, May 2017.

[9] M. Z. Hassan, V. C. M. Leung, M. J. Hossain, and J. Cheng, "DelayQoS aware adaptive resource allocations for free space optical fronthaul networks," in Proc. GLOBECOM, Singapore, Dec. 2017, pp. 1-6.

[10] K. P. Peppas and P. T. Mathiopoulos, "Free-space optical communication with spatial modulation and coherent detection over H-K atmospheric turbulence channels," J. of Lightw. Technol., vol. 33, no. 20, pp. 42214232, Oct. 2015.

[11] M. Jeganathan and K. Kiasaleh, "Transceiver, system, and method for free-space optical communication and tracking," Sep. 19 2002, uS Patent App. 09/808,496.

[12] A. A. Farid and S. Hranilovic, "Outage capacity optimization for freespace optical links with pointing errors," J. of Lightw. Technol., vol. 25, no. 7, pp. 1702-1710, Jul. 2007.

[13] M. Avriel, W. E. Diewert, S. Schaible, and I. Zang, Generalized concavity. New York: Plenum: SIAM, 2010.

[14] A. D. Belegundu and T. R. Chandrupatla, Optimization Concepts and Applications in Engineering. Cambridge University Press, 2011.

[15] R. Laha, "On a class of unimodal distributions," Proc. of the American Mathematical Society, vol. 12, no. 2, pp. 181-184, Apr. 1961. 